\title{
Modified participation ratio approach: application to edge-localized states in carbon nanoclusters
}

\author{
A.V.Luzanov \\ SSI "Institute of Single Crystals", National Academy of Sciences of \\ Ukraine, 60 Nauky Ave., 61078 Kharkiv, Ukraine
}

Received April 7, 2016

For nanoclusters and solids, the localization analysis of one-electron states, or MOs (molecular orbitals), is frequently provided by using the so-called participation ratio (PR) index. To this conventional PR approach, we add for each MO the new index $\sigma_{\text {IPR }}$ which we define as an average fluctuation of the inverse PR (IPR) value. Typically, the $\sigma_{\text {IPR }}$ index displays a significant sensitivity to any spatial irregularity in the MO distribution over molecule. We apply the thus extended PR analysis to the graphene nanoflakes of different types, and small nanodiamond structures including NV color centers as well. The proposed scheme has the virtue of being quite simple, and in case of huge clusters it allows one to rapidly detect orbitals with unusual non-uniform distribution. In particular, the localization of edge states in graphene molecules is examined in this way.

Keywords: participation ratio, edge localization, graphene nanoflakes, nanodiamonds, NV centers.

Для нанокластеров и твердых тел анализ локализации одноэлектронных состояний, или МО (молекулярних орбиталей), часто осуществляют с помощью так называемого индекса отношения участия (PR). В этот подход мы добавляем для каждой МО значение нового индекса $\sigma_{\mathrm{IPR}}$, который определяем как некую среднюю флуктуацию обратного индекса PR (IPR). Данный $\sigma_{\mathrm{IPR}}$-index демонстрирует значительную чувствительность к какой угодно нерегулярности в распределении MO по молекуле. Расширенный таким образом PR-анализ применен к графеновым наночешуйкам различной природы и к небольшим наноалмазным структурам, включающим также NV-центры окраски. Предложенная схема, как достаточно простая в работе вполне әффективна - она позволяет быстро находить орбитали с непривычно большой неоднородностью распределения в больших кластерах. В частности, таким образом изучена локализация краевых состояний графеновых молекул.

Модифікований підхід відношення співучасті: застосування до крайових станів вуглецевих нанокластерів. А.В.Лузанов.

Для нанокластерів та твердого тіла аналіз локалізації одноелектронних станів, або MO (молекулярних орбіталей), часто запроваджують за допомогою так званого індексу відношення співучасті (PR). До цього підходу ми додаємо для кожної МО значення нового індексу $\sigma_{\text {IPR }}$, котрий визначаємо як деяку середню флуктуацію оберненого індексу PR (IPR). Цей $\sigma_{I P R}$-index демонструє значну чутливість щодо будь-якої нерегулярності у розподілі MO вздовж молекули. Розширений таким чином PR-аналіз застосовано до графенових нанолусок різних типів та до малих нанодіамантових структур, що включають також NV-центри забарвлення. Як цілком проста у роботі, запропонована схема є досить ефективною - вона дозволяє швидко відшукувати орбіталі з незвичним неоднорідним розподілом у величезних кластерах. Зокрема, в такий спосіб вивчено локалізацію крайових станів графенових молекул. 


\section{Introduction}

Electron localization analysis for molecules and solids is well addressed in the vast literature. Among the methods used, the so-called participation ratio (PR) plays important role, in particular for studying Anderson localization of electron states in disordered systems [1,2]. In a simplest case, $P R$ is defined as

$$
\mathrm{PR}=1 / \sum_{\mu}\left|c_{\mu}\right|^{4}
$$

In Eq. (1) $c_{\mu}$ are usual expansion coefficients for the investigated state which is given in terms of atomic orbitals (AOs) or other basic functions. The PR index can be considered as a number of the most important basis functions involved in the state under study. The localization (more exactly, delocalization) measure of this kind was first proposed in [3] for interpreting normal vibrational modes in crystals. Independently, the analogous quantity, called the collectivity number, was introduced in [4] for treating amplitudes in the single excitation configuration interaction scheme (see also [5]).

PR and IPR (inverse partition ratio) are rather widely used in various quantum-mechanical problems. For instance, IPR naturally appears in the quantum transition theory for analyzing survival probabilities (see p.77 in [6]). At the same time, using PR for electron localization in molecular systems is not so frequent (except for the known orbital-localization theory given by Pipek and Mezey [7]). In this light, the main motivation for the present work is to extend applicability of the PR technique to orbital localization phenomena in large molecules. In the latter, localization phenomena should be more interesting and more diverse than in small-size systems. That this is the case can be easily understood by instances from condensed matter physics. For example, Tamm put forward the fundamental idea of surface states which may be present on the surface due to a termination of the bulk crystal [8]. The subsequent extension and development of the Tamm surface states theory was thoroughly discussed in the well-known reviews [9, 10] and in book [11]. It is interesting to study in detail similar quasi-surface states which possibly occur in nanocrystals and related finite-size atomic clusters. However, only a few works on this topic can be found in the literature [12-19].
Generally, the consistent edge ( surface) state theory is too involved - in concordance with the Pauli sentence: "God made solids, but surfaces were the work of the devil". In particular, analytical solutions of edge states were derived within simple tight-binding approaches only for few examples of finite nanotubes and nanographenes $[16,17]$. Notice that even in the current literature, a quantitative characterization of surface-state localization is rarely provided.

Continuing our recent work about electronic properties of nanographenes and nanodiamonds $[20,21]$, the present paper aims to investigate edge-like states by appropriate localization measures. To deal with large-scale structures, such as huge conjugated and diamondoid carbon-cage systems (condensed adamantanes) we will mainly exploit simple Huckel-type approximations. In so doing, we propose an ancillary interpretation index which helps screening and analyzing large orbital arrays. A brief sketch of the paper is as follows: The main localization measures are given in section 2. The additional characteristic (our index $\sigma_{\text {IPR }}$ ) is proposed in section 3. Applications to carbon nanomaterials (graphene nanoflakes and nanodiamonds) are shown in sections 4 and 5. Miscellaneous problems of orbital localizations are presented in section 6 , and the last section concludes the paper. In Appendix we discuss the interplay of the edge localized states and effectively unpaired electrons.

\section{Main measures of orbitals localization}

To begin, we describe more or less familiar localization measures. The usual localization measure of the given MO (molecular orbital) is a sum of the selected squared LCAO (linear combination of AOs) coefficients. Throughout the paper we assume a common AO representation of MOs which can be, by and large, obtained numerically. Moreover, only canonical MOs (eigenstates of effective one-electron Hamiltonians) and relevant natural orbitals will be studied in this paper. Thus, our subject here is not artificially localized non-canonical MOs (which, however, can be particularly suitable for configuration-interaction methods).

Before returning to PR index (1), we make some additional notational definitions. Let $\left|\varphi_{j}\right\rangle$ be the MO in question. We bring in the standard LCAO expansion of the form 


$$
\left|\varphi_{j}\right\rangle=\sum_{\mu} c_{\mu j}\left|\chi_{\mu}\right\rangle
$$

where the appropriate orthonormal AO basis

$$
\left\{\left|\chi_{\mu}\right\rangle\right\}_{1 \leq u \leq \text { dim }}
$$

is employed, and dim is a size of the basis used. LCAO coefficients $c_{\mu j}$ in Eq. (2) are obviously interpreted as elementary probability amplitudes. Then the set

$$
\left|c_{\mu j}\right|^{2} 1 \leq \mu \leq \operatorname{dim}
$$

produces probabilities of finding electron on corresponding AOs or corresponding atomic centers. It allows one to assign to each MO $\varphi_{j}$ the ordinary localization measure for the selected molecular fragment $A$ :

$$
L_{A}\left[\varphi_{j}\right]=\sum_{\mu \in A}\left|c_{\mu j}\right|^{2} .
$$

For the given MO $\varphi_{j}$, we will term the set of indexes $\left\{L_{A}\left[\varphi_{j}\right]\right\}$ the atomic distribution if $A$ in Eq. (4) is reduced to atom. Along with these commonly used quantities, other additional measures characterize integrally oneelectron state distribution over the whole molecule. One of these is the above mentioned participation (or partition) ratio PR. For the $j$ th probability distribution (3), this index is

$$
\mathrm{PR}=1 / \sum_{\mu}\left|c_{\mu j}\right|{ }^{4} .
$$

giving a good measure of the number of AO or atomic centers which effectively participate in MO $\varphi_{i}$.

For what follows we need some more notation. Let

$$
w=\left\{w_{k}\right\}_{1 \leq \mu \leq \operatorname{dim}}
$$

denote a probability set of nonnegative weights normalized to unity. Then, the PR index for $w$ is of the form:

$$
\mathrm{PR}=1 / \sum_{k=1}^{\operatorname{dim}} w_{k}^{2} .
$$

It is also possible to present more general indexes [22]. For our purposes, the most important is the second order PR index

$$
\text { PR2 }=\left[\sum_{k}\left(w_{k}\right)^{2}\right]^{2} / \sum_{k}\left(w_{k}\right)^{4} .
$$

We may expect, as in [22] for another problems, that Eq. (8) should provide a more sharp estimate for an effective number of atomic centers with strong orbital localization. Helpful illustrations will be considered later.

\section{Modified localization analysis}

\subsection{General expressions}

Now we introduce our ancillary index $\sigma_{\text {IPR }}$ which helps us to quickly fix significantly localized MOs in large-scale orbital arrays. We turn attention to the fact that in Eqs. (7) and (8) we deal with "inner" averages of the form

$$
\left\langle w^{k}\right\rangle=\sum_{k} w_{k}\left(w_{k}\right)^{k}
$$

where $\left.\left(w_{k}\right)^{k}\right)$, the $k$ th powers of probabilities, serve as random quantities to be usually averaged. In these terms, localization index (7) is of the form

$$
\mathrm{PR}=1 /\langle w\rangle .
$$

Analogously, Eq. (8) is equivalent to

$$
\mathrm{PR} 2=\langle w\rangle^{2} /\left\langle w^{3}\right\rangle,
$$

and, the frequently employed IPR index is the simplest average of the form

$$
\operatorname{IPR}=\sum_{k}\left(w_{k}\right)^{2}=\langle w\rangle .
$$

Eq. (12) suggests an idea to bring into play $\sigma=\left\langle w^{2}\right\rangle-\langle w\rangle^{2}$, the variance of the probability distribution itself. In practice, we will make use the scaled standard deviation

$$
\sigma_{\text {IPR }}=\operatorname{dim}\left(\left\langle w^{2}\right\rangle-\langle w\rangle^{2}\right)^{1 / 2} .
$$

The scale prefactor, dim, in Eq. (13) provides more suitable non-disappearing values of the index even for very large basis sizes, $\operatorname{dim}>>1$.

We shortly explain why the variance can be useful for analyzing localization. By construction, the variance describes fluctuations about the expected value. It means in our case that $\sigma^{2}$ reflects a nonuniformity of the distribution under study. Clearly, the maximal delocalized states ( $w_{j}$ equiv $\left.1 / \mathrm{dim}\right)$ provide the most uniform (flat) distribution with minimal variance $\sigma^{2}=0$. On the contrary, essentially localized states produce highly non-uniform distributions with a high variance. This makes $\sigma^{2}$ (or $\sigma_{\text {IPR }}$ ) a good indicator of localization. Thus, in the present study we will mainly utilize delo- 
calization measure PR2 and $\sigma_{\text {IPR }}$ index, which are defined by Eqs. (11) and (13), respectively. The $\sigma_{\text {IPR }}$ index will be termed the fluctuation index. To understand how it works in reality we explore some examples.

3.2. Using localization indexes for dimerized $1 D$ chain

A finite monatomic chain with one electron per atom gives us the system which is simple enough to be tractable, and complicated enough to contain edge localization phenomena. The standard tight-binding (TB) approximation with neglecting orbital overlap (Huckel method) will be used here. In fact, we will consider the $\pi$-electron structure of a polyene chain with $N$ carbon atoms. The basic TB Hamiltonian parameters of the chain are the so-called resonance (hopping) integrals $\beta_{\mu, \mu+1}(1 \leq \mu \leq N-1)$ between nearest neighboring sites. In units of the standard (negative) hopping integral $\beta_{0}$, they are nonnegative and can be taken as follows: $\beta_{\mu, \mu+1}=1$ for odd $\mu$, and $\beta_{\mu, \mu+1}=1-\eta$ for even $\mu$, where $\eta$ is an alternation parameter. In this notation, $N=\operatorname{dim}$ (a number of the used AOs), and the well-known Lennard-Jones result [23] for Huckel orbital energies of the chain is as follows:

$$
\varepsilon_{j}= \pm\left[1+(1-\eta)^{2}+2(1-\eta) \cos 2 k_{j}\right]^{1 / 2},(14)
$$

where, for sufficiently long chain we have approximately $k_{j}=\pi j / N+1$, and $1 \leq j \leq N$. This is the case of a dimerized Peierls insulator without defects.

In the case of a fully regular (homogeneous) chain when $\eta=0$ (no dimerization), we have $\varepsilon_{j}=\varepsilon_{j}= \pm 2 \cos k_{j}$, and the corresponding LCAO coefficients are

$$
c_{\mu j}=\left(\frac{2}{N+1}\right)^{1 / 2} \sin \mu k_{j} .
$$

For this case, the computation of the main localization indexes is easily performed analytically; the required sums of powers of $\sin k_{j}$ are well know [24]. More convenient are asymptotical expressions which can be simply computed by integrals given also in [24], entry 3.621.3, The final result, which is valid for any MO of the homogeneous chain, is

$$
\left\langle w^{k}\right\rangle=(2 N)^{-k}\left(\begin{array}{l}
2 k+2 \\
k+1
\end{array}\right) / 2,
$$

so we have

$$
\begin{aligned}
\mathrm{PR}=2 N / 3, \mathrm{PR} 2 & =18 N / 35 \cong 0.413 N,(17) \\
\sigma_{\mathrm{IPR}} & =1 / 2,
\end{aligned}
$$

for each MO of the form (15). The same results hold for the $1 \mathrm{D}$ particle in a box problem (then $N$ in Eq. (17) should be replaced by the box length).

We see that, as it should be in this case, all MOs are the delocalized (extended) states having a relatively small value of fluctuation index (13). Thus, the homogeneous chain does not show any anomalous orbitals (all states are extended). It is interesting that in the limit as $N \rightarrow \infty$, the same Eqs. (17) are approximately true for the frontier MOs in the slightly dimerized chain $(\eta \neq 0)$.

A more interesting picture occurs if the selected $\beta_{\mu, \mu+1}$ is perturbed. This problem was thoroughly studied in the analytical manner in [25]. In this it was investigated under which conditions the local levels of various types appear. In particular, in case of the dimerized chain (then $\eta \neq 0$ ) the edge state in the forbidden band is possible if the terminal hopping integral $\beta_{\text {edge }} \equiv \beta_{1,2}$ satisfies the inequality:

$$
\beta_{\text {edge }} \leq \eta
$$

With this in mind, let us perform numerical simulations. We consider the dimerized chain with $N=100$, and the fixed $\eta$ value, $\eta=0.25$. Let us examine two cases: $\beta_{\text {edge }}=1$ (the usual Peierls insulator), and $\beta_{\text {edge }}=\eta=0.25$ (the same insulator with the embryonic edge state). A summary of the results can be found in Table 1 . In it, $L_{\text {edge }}^{\mathrm{HOMO}}$ is $\left(c_{1}\right)^{2}$ for the terminal carbon atom $\mu=1$ in case of the highest occupied MO (HOMO). We see that indeed in the normal Peierls chain, the delocalization of MOs is almost the same as in the regular chain [compare, e.g., the corresponding maximal $\sigma_{\text {IPR }}$, $\sigma_{\text {IPR }}^{\max }$, in Table 1 and $\sigma_{\text {IPR }}$ in Eq. (18)]. The situation is sharply different when $\beta_{\text {edge }}=\eta$, and an edge state appears. Then from $\sigma_{\text {IPR }}$ plot vs orbital number $j$ (i. e., vs eigenstate index) we observe that for HOMO the fluctuation index increases more than order: $\sigma_{\mathrm{IPR}}^{\max }=\sigma_{\mathrm{IPR}}^{\mathrm{HOMO}} \cong 18$. It corresponds to the case of a strong edge localization of the frontier state as it must be according to [25]. Thus, $\sigma_{\text {IPR }}$ plot helps us simply and easily to diagnose the localized nature of the state independently of the values of 
Table 1. The squared HOMO Huckel coefficients $\left(c_{\mu}\right)^{2}$; delocalization indexes $\mathrm{PR} 2_{\text {HOMO }}$; fluctuation index $\sigma_{\mathrm{IPR}}$; and $L_{\text {edge }}^{\mathrm{HOMO}}$ in the 100 -carbon dimerized polyene chain with $\eta=0.25$

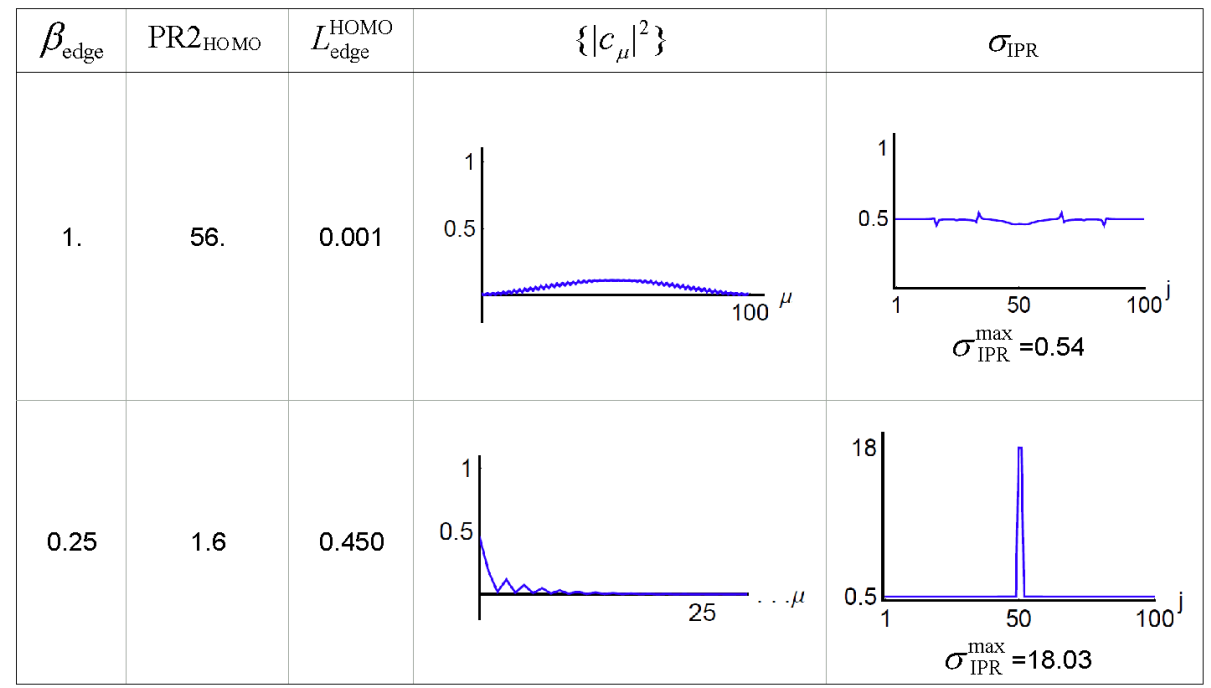

other indexes. Such an easiness is the essential merit when very large amount of orbitals must be handled, as it occurs in case of huge molecular systems and atomic clusters which we consider next.

\section{Edge localization of frontier MOs in nanographenes}

Recall that two types of edges are usually recognized in the graphene nanoclusters: armchair and zigzag edges. The armchair edges contain phenanthrene-like units, whereas the zigzag edges contain anthracene-like units. Peculiarities of the orbital localization in large graphene-like structures are discussed in much work (e.g., see [26-30]). In particular, as established first in [26], and later in [27], the frontier MOs are essentially localized on zigzag edges. Significantly, several experiments [31-33] have provided excellent supporting evidences in favor of localized edge states in graphene nanoribbons.

Here we study quantitatively the Huckel $\pi$-orbital localization in several graphenelike molecules (graphene nanoflakes) presented in Table 2. For the sake of brevity, we will use shorthand $C_{N}$ in dealing with graphene molecule having $N$-carbon atom backbone which is terminated at the periphery by hydrogen atoms. We take almost the same examples which were previously investigated [20, 21, 34]. The full edge localization index $L$ edge is computed for HOMO by standard Eq. (4) where only edge carbon atoms with degree 2 (number of neighboring carbons are taken into account in the respective sum over $\mu$. Furthermore, in Tables we display for each system its skeletal formula on which is superimposed the atomic distributions of the corresponding HOMO. Notice that the carbon backbone of graphene molecules belongs to the class of the so-called alternant systems (bipartite graphs) for which the Coulson-Rushbroke orbital parity theorem is valid. Then orbital localizations (4) of the given occupied MO and dual virtual MO are the same because

$$
\left|C_{\mu j}\right|^{2}=\left|C_{\mu, \operatorname{dim}-j+1}\right|^{2}
$$

That is why in the tables relating to alternant $\pi$-structures we give $\sigma_{\text {IPR }}$ plots vs orbital number only for the occupied MO's set.

In this regard, notice a technical point concerning $\pi$-orbital degeneracy in the studied hexagonal honeycomb structures with $D_{6 h}$ symmetry. For these and any other systems involving as well an accidental, or hidden, symmetry, a counterpart of probability set (3) should be computed by taking into account all partner orbitals of the corresponding degenerate representation. If ignoring this point, an irrelevant asymmetry is involved, thereby making artificially more localized MOs, and markedly overestimated localization indexes. However, in the literature this point is not duly discussed. In particular, we do not forget about a possible accidental degeneracy for Huckel orbital energies $\varepsilon_{j}=1$ (and for $\varepsilon_{j}=$ -1 as well). This degeneracy is incidental to 
Table 2. HOMO localization indices $L_{\text {edge }}^{\mathrm{HOMO}}, \mathrm{PR} 2_{\mathrm{HOMO}}$, and plot $\sigma_{\mathrm{IPR}}$ vs orbital number $j$ for typical graphene nanoflakes

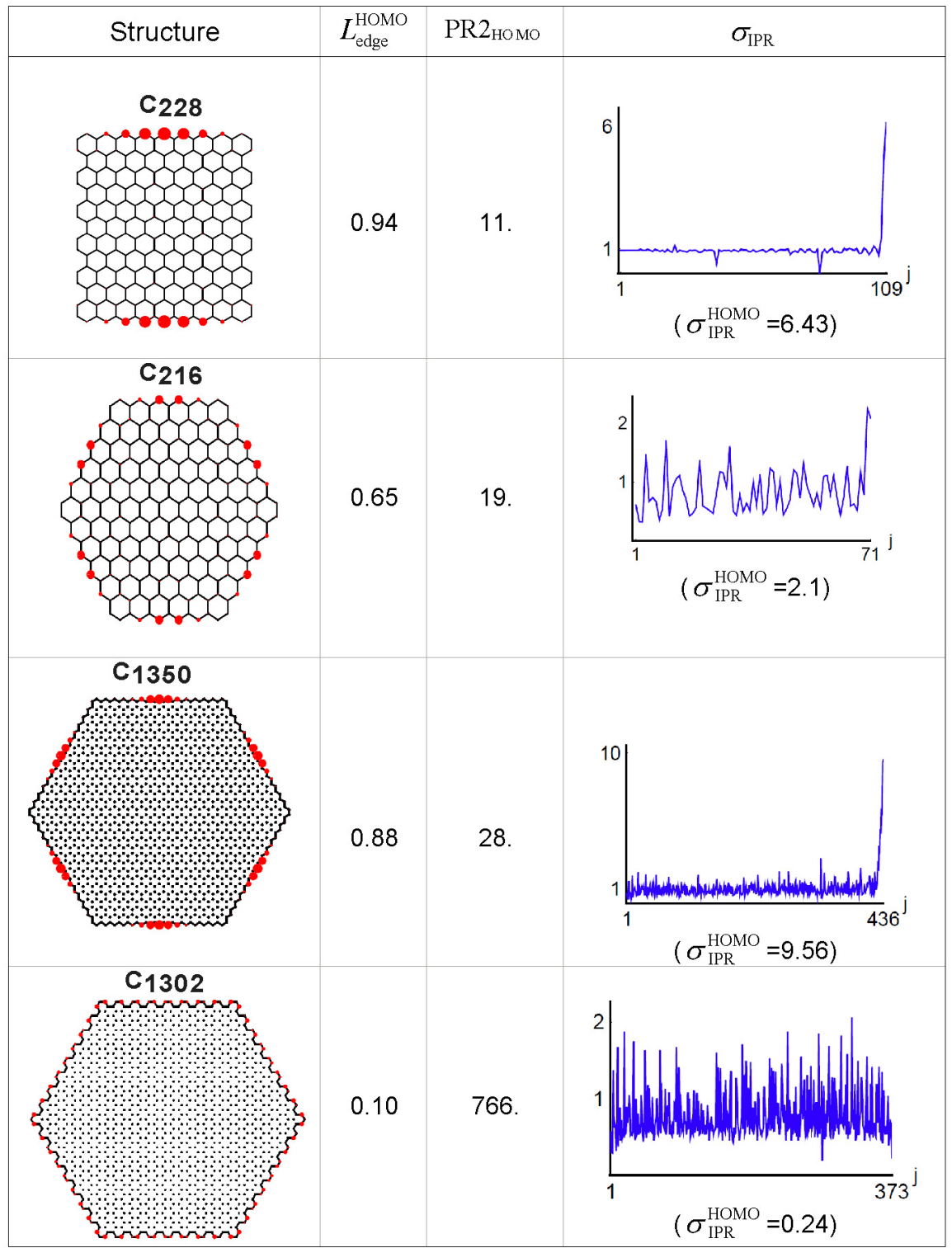

many graphene $\pi$-structures. On this account, for most of the graphenes given in Table 2 the actual number $N_{\text {occ }}$ of occupied $\pi$-MOs does not coincide with $\operatorname{dim} / 2$.

We first consider a small nanoribbon $\mathrm{C}_{228}$ with horizontal zigzag edges. From the $L$ edge values and the corresponding pictorial images, the above-mentioned strong localization on the zigzag edges is quite evident. In $\mathrm{C}_{228}$ and other systems, delocalization index PR2 provides a reasonably crude estimation of numbers of the most active atoms contributed to HOMO, as clear from the images of Table 2. Besides, $\sigma_{\text {IPR }}$ plots furnish an additional useful information characterizing all MOs. From these plots in Table 2 we see that in all the cases, $\sigma_{\text {IPR }}$ attains its maximal value just for HOMO (and thus for LUMO, lowest unoccupied MO). These $\sigma_{\text {IPR }}^{\mathrm{HOMO}}$ values are significantly larger than 0.5 (compare with corresponding the small value in Eq. (18) for the fully delocalized HOMO of the infinite polyene chain). It fixes the high localization of HOMO/LUMO states in zigzag-edge graphene nanoribbons without invoking pictorial images; additionally it assures the absence of another essentially local states. Notice also that $\sigma_{\text {IPR }}$ plot can detect possible 


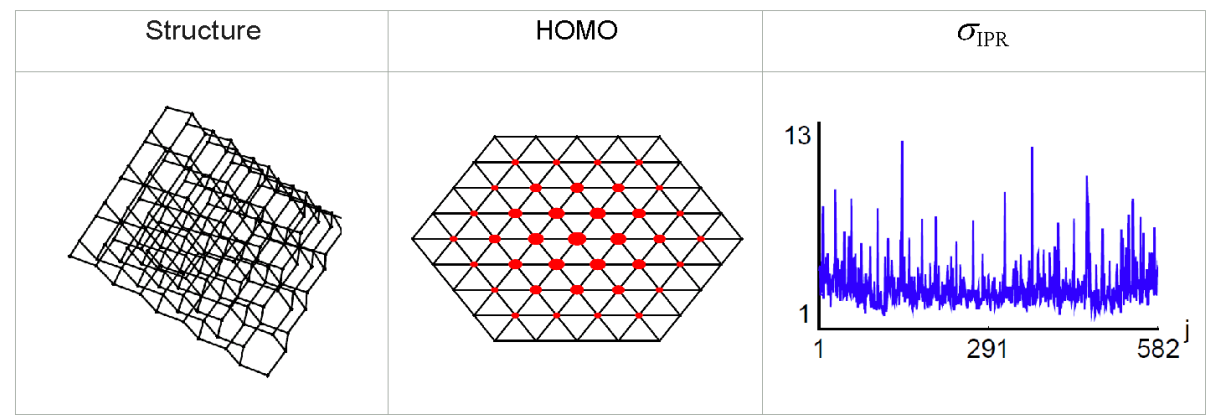

Fig. 1. Structure, HOMO localization diagram, and $\sigma_{\text {IPR }}$ plot for diamondoids $\mathrm{C}_{190} \mathrm{H}_{110}$.

localization in any region of the molecule (not only the edge localization).

Some additional words about the hexagonal nanoflakes $\mathrm{C}_{216}$ and $\mathrm{C}_{1350}$. Table 2 shows, for HOMO's, the same preferable localization on zigzag border atoms. Concurrently, we note that cluster $C_{1302}$, having the perfect armchair edges, produces, as expected, small values of the HOMO characteristics $L_{\text {edge }}^{\mathrm{HOMO}}$ and $\sigma_{\mathrm{IPR}}^{\mathrm{HOMO}}$. The latter are fully consistent with a very large value of the delocalization index PR2 (the 3rd column in the Table).

\section{Searching edge states in small nanodiamonds}

Here we discuss the orbital localization for another interesting class of carbon nanomaterials; namely those of diamondlike structure. These are nanodiamondoids, which represent a group of $\mathrm{sp}^{3}$ carbon-cage molecules with dangling carbon bonds normally passivated by $\mathrm{H}$ atoms. These systems generate very important materials with broad spectrum of properties and applications [35-37].

The nanodiamond structure of the formula $\mathrm{C}_{190} \mathrm{H}_{110}$ (related systems were studied previously in [38]) will serve here as the main illustrative example. We employ the so-called extended Huckel method of the conventional type [39]. The geometric image of the cluster ( $\mathrm{H}$ atoms are suppressed) is given in the left panel of Fig. 1. The central panel of the figure represents the HOMO atomic distribution which is displayed making use a suitable plane projection of the molecule. We see that HOMO is preferably localized inside the carbon cage in agreement with a small value of the surface localization index: $L_{\text {edge }}^{\mathrm{HOMO}}=0.226$, that is the HOMO is approximately $76 \%$ bulk orbital. The modest magnitude of $\sigma_{\{} \mathrm{IPR}^{\mathrm{HOMO}}=3.53$ and $\mathrm{PR} 2_{\mathrm{HOMO}}=45.7$ are in concordance

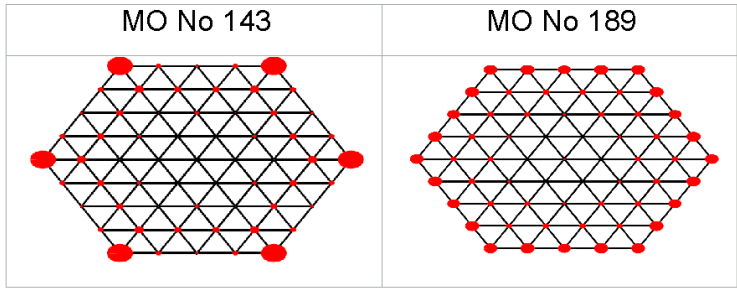

Fig. 2. Localization diagrams for special MOs with orbital numbers 143 and 189 in diamondoid $\mathrm{C}_{190} \mathrm{H}_{110}$.

with a moderately delocalized character of this bulk orbital. Incidentally we must remark that in the used projections, the plane images of several atoms are superimposed; but in our cases this does not prevent observing orbital localizations clearly.

From $\sigma_{\text {IPR }}$ plot in Fig. 1 we can also expect the existence of more interesting orbitals with highly inhomogeneous atomic distribution. For instance, what are the properties of the MO giving in $\mathrm{C}_{190} \mathrm{H}_{110}$ the maximum value $\sigma_{\mathrm{IPR}}^{\max }=12.65$. We find that this orbital has eigenstate number 143, and respectively, $L_{\text {edge }}^{[143]}=0.60$; the corresponding atomic distribution is displayed in the left panel of Fig. 2. Searching the orbital with maximum edge, i. e., is surface localization (its eigenstate number is 189) provides somewhat different picture (see the right panel of Fig. 2): a more homogeneous distribution over the surface (edge) atoms, and $L[189]=0.742$. Respectively, no more than a moderate magnitude $\sigma_{[\mathrm{PR}}^{[189]}=3.67$ is produced.

Yet, another picture for nanodiamonds with interior point defects. In Fig. 3 we present the images for HOMO and LUMO in the case of $\mathrm{NV}^{-}$(negative nitrogen-vacancy) color center generated in the cluster $\mathrm{C}_{190} \mathrm{H}_{110}$. In this case $\sigma_{\mathrm{IPR}}^{\max }=60.3$, and this sharp maximum (see $\sigma_{\text {IPR }}$ plot in Fig. 3) is 


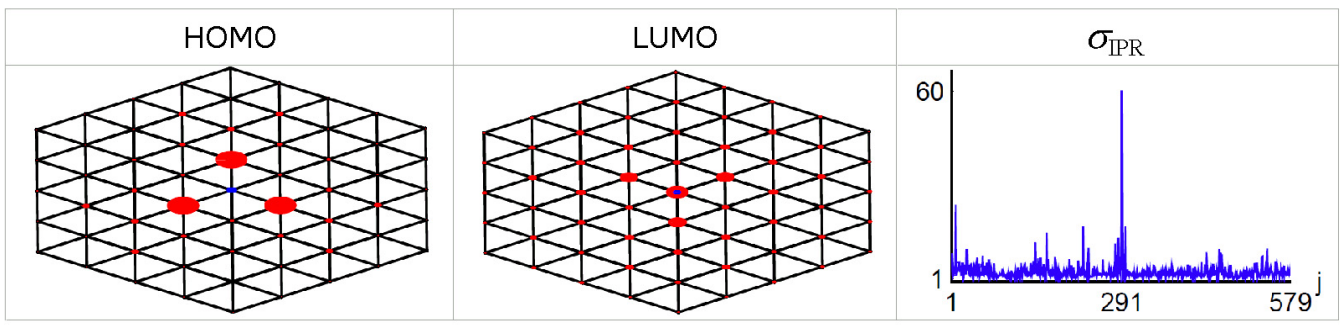

Fig. 3. MO localization properties of the simulated $\mathrm{NV}^{-}$center $\mathrm{C}_{188} \mathrm{H}_{110} \mathrm{~N}^{-}$.

Table 3. Atomic distribution of active MOs in functionalized finite-size graphene $\mathrm{C}_{238}$

\begin{tabular}{|l|l|l|}
\hline HOMO & LUMO & $\sigma_{\mathrm{IPR}}$ \\
\hline & & \\
\end{tabular}

exactly attained at the HOMO, if treating an artificial singlet closed-shell state of the cluster. (Peculiarities of spin states of $\mathrm{NV}$ centers are not the subject of this paper; for this issue see discussion and references in $[20,40])$. It is worth noting that judging from Fig. 3, in the used model, HOMO is markedly more localized in a vicinity of the color center than the corresponding LUMO (the projected position of $N$ atom is indicated in Fig. 3 by a small blue circle). On the whole, the localization of these HOMO and LUMO is familiar to the localization of the of $\mathrm{NV}^{-}$-center lowest excitations which was previously given in [40]. This fact seems quite natural since the frontier MOs are normally the most important in forming lowest excitations.

\section{Modified problems}

Here we return to graphenes and discuss additional problems in which we vary the type of the nanographene borders and make other modifications. Functionalized, in particular edge-modified nanographene is a common and current theme in the graphene literature [37, 41-44]. We consider now only one kind of the edge modification. By making on armchair edges the cyclization of peripheral phenanthrene subunits we replace them by fluorenyl subunits, as it is shown in structure $\mathrm{C}_{238}$ from Table 3 . This system is no longer alternant one, and symmetry relations (20) between occupied and virtual $\pi$-MOs are violated. Accordingly, we display in the Table the localization diagram for the both frontier MOs: HOMO and LUMO. From $\sigma_{\text {IPR }}$ plot we see that $\sigma_{\text {IPR }}$ corresponds, not to the HOMO, but to a more deep orbital, HOMO-15 in fact. For completeness, we show in Table 3 the HOMO-15 localization diagram, too. Comparing all three orbital images given in the Table we observe that HOMO is now a lesser localized than the other non-frontier MOs, and just HOMO-15 (having the maximum value of $\bar{\sigma}$ ) turns out to be to well localized on the modified armchair (not zigzag!) edges. Remark that in this case the detection of the HOMO-15 localized on the modified armchair edges, has become possible owing to a direct simple search of $\sigma_{\mathrm{IPR}}^{\max }$ in the full set of orbital fluctuations. 


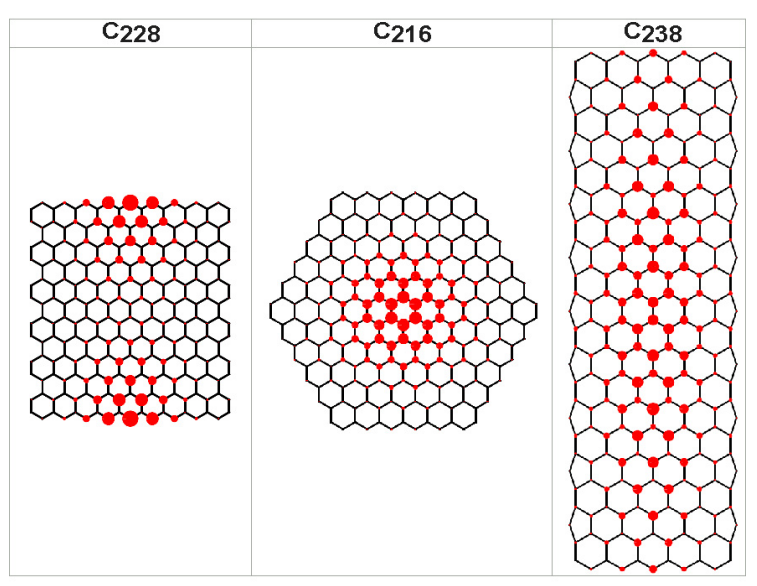

Fig. 4. Localization diagrams of the Huckel HOMO for selected graphene nanoflakes in the strong magnetic field.

Let us now examine the same system $\mathrm{C}_{238}$ and others in a strong atomic-scale magnetic field. The calculations are performed in framework of the conventional Huckel-London theory which is based on using gauge-invariant $\pi$-AOs [45]. For testing the strong field effect we took the static field strength 0.02 a.e., at which quite appreciable changes in the HOMO properties occur (Fig. 4). The most interesting effect is that the strong field tends to localize the HOMO in the molecule's interior in such a way that the edge localization becomes weaker (as in $\mathrm{C}_{228}$ ), and even disappears (as in $\mathrm{C}_{216}$ ). As a result, the edge magnetism must vanish in strong magnetic fields.

At last, the important issue which deserves at least a short consideration is the effects of methods used on the results. In the illustrations hitherto given, we employed the Huckel-type methods as a simplest model providing qualitatively reasonable orbital picture. The recent paper [46] provides an interesting insight on the impact of Huckel-based models in an ab initio era. Stress that the Huckel method and its variations (tight-binding techniques) are a starting point of conventional theoretical approaches to the solid-state physics of graphene. Nonetheless, more refined approaches can generally lead to a somewhat different orbital localization, especially in large-scale systems.

To somehow understand the situation we invoke here the MOs which are produced by the unrestricted Hartree-Fock (UHF) method within the conventional PariserParr-Pople semiempirical $\pi$-scheme $(\pi$-UHF). For these UHF calculations we take the
Table 4. Comparison of the $\pi$-UHF, pseudo$\pi$ UHF, and QCTB values for HOMO and EUE localization indexes in $\mathrm{C}_{78}$

\begin{tabular}{|c|c|c|c|}
\hline 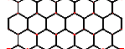 & $\pi-U H F$ & Pseudo-T UHF & QCTB \\
\hline PR2 & 7.0 & 8.1 & 7.8 \\
\hline$\sigma_{\mathrm{IPR}}^{\mathrm{HOMO}}$ & 3.36 & 2.87 & 3.01 \\
\hline$N^{\mathrm{EUE}}$ & 4.71 & 4.45 & 5.25 \\
\hline $\mathrm{PR} 2^{\mathrm{EUE}}$ & 11.4 & 13.8 & 16.2 \\
\hline$L_{\text {edge }}^{\text {EUE }}$ & 0.642 & 0.670 & 0.586 \\
\hline
\end{tabular}

carbon backbone formed by regular hexagons with the $\mathrm{C}-\mathrm{C}$ bond length of $1.4 \AA$. We should notice that for any UHF models there is a difficulty in using the canonical MOs generated by the standard Fock operators $f_{\alpha}$ and $f_{\beta}$ of spin-up and spin-down electrons, respectively. In general, these orbitals, taken separately, do not display the full molecular symmetry even though the total UHF wave function is in agreement with the required symmetry [47] (if ignoring possible symmetry breaking). Unlike such UHF MOs, the associated natural orbitals are symmetry-adapted ones. Thus, we must compare the Huckel MOs just with these natural orbitals.

In addition to $\pi$-UHF, we employ the socalled pseudo- $\pi$ model which was introduced in [48] to mimic ab initio magnetic $\pi$-shell properties in large polyaromatic hydrocarbons (PAHs). In this model, instead of the given $\mathrm{PAH}$ one takes a purely hydrogen cluster (H-cluster) with the geometry of the corresponding carbon backbone, and does the Hartree-Fock computation of the H-cluster at the STO-3G level. However, the $\pi$ electron correlation effects would be overestimated if one applies this model within the post-Hartree-Fock methods. At the same time, using smaller bond lengths enables correlation effects to be reasonably reduced. Here we introduce just this modification of the pseudo- $\pi$ model with the reduced neighbor $\mathrm{H}-\mathrm{H}$ length values all equal to $1 \AA$. In practice, we simply scale the $\mathrm{PAH}$ backbone Cartesian coordinates in by a factor of $1 / 1.4$.

The typical are the results obtained for the localization indices in nanocluster $\mathrm{C}_{78}$ (Table 4). We compare the HOMO properties obtained from QCTB [21,34] (the Huckellike model sketched in Appendix) with that of the HOMO counterparts produced by $\pi$ UHF and the above-mentioned pseudo- $\pi$ 
Table 5. Number of effectively unpaired electrons $N^{\mathrm{EUE}}$, delocalization index PR2 ${ }^{\mathrm{EUE}}$, distribution of EUE over the carbon backbone, and plot $L_{\text {edge }}$ vs orbital number $j$ for representative graphene nanoflakes

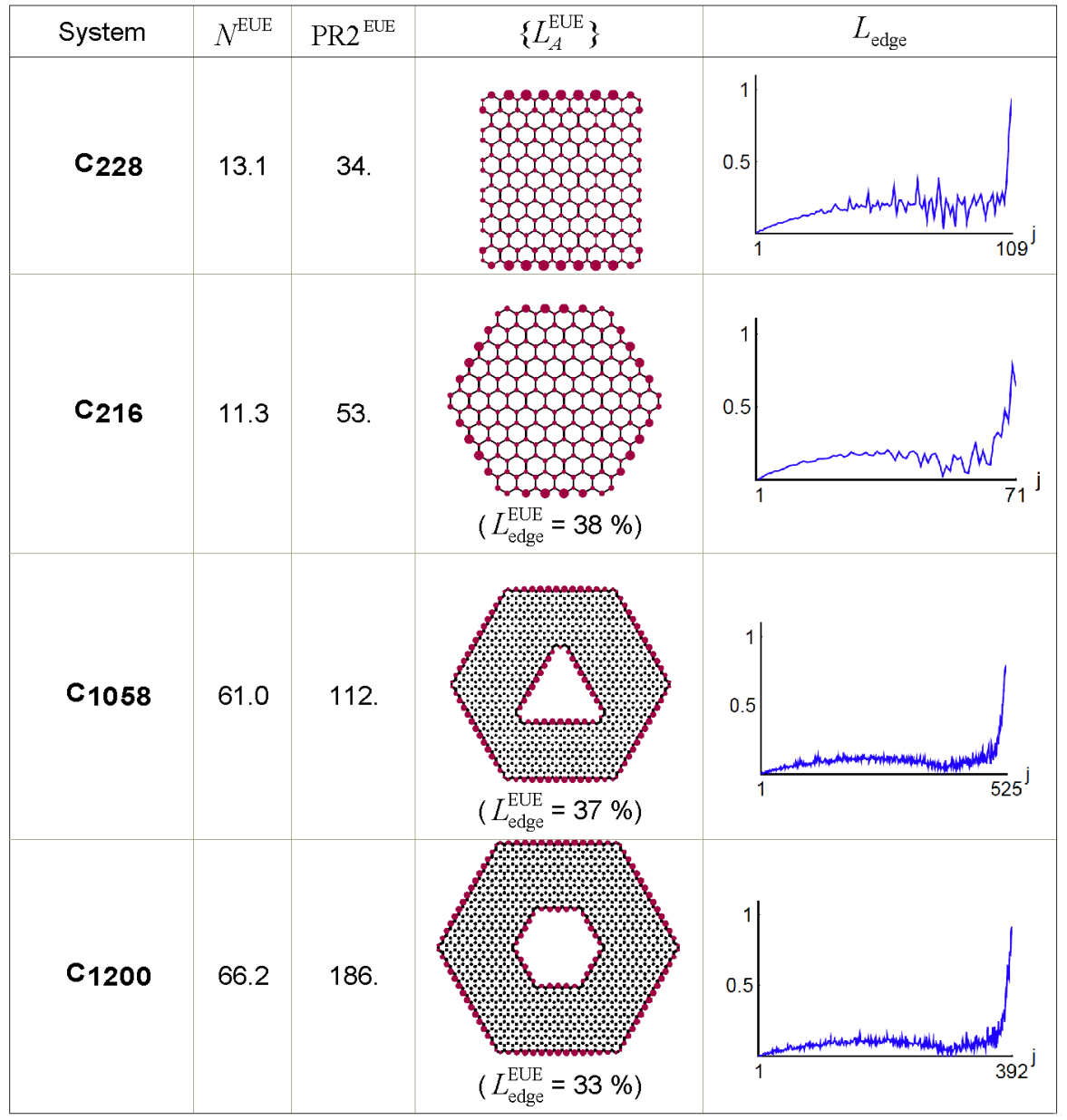

model at the UHF level (pseudo- $\pi$ UHF in Table 4). Moreover, the characteristics of effectively unpaired electrons (EUE, see Appendix), are also presented in the Table. We see that on the whole, all the used schemes provide a qualitatively similar picture: the strong edge localization of HOMO and the well localized (preferably on zigzag edges) unpaired electrons. Most noticeable is a difference in quantitative EUE characteristics. We observe that the applied, rather crude QCTB scheme overestimates the $N^{\mathrm{EUE}}$ index, that is the EUE number (see Eq. (A1) in Appendix). The same is true for the delocalization character of the unpaired electrons as well (compare the PR2 ${ }^{\text {EUE }}$ values in Table 5).

\section{Concluding remarks}

In this paper, the PR analysis of orbital localization is discussed and supplemented with the new index $\sigma_{\text {IPR, Eq. (13). Using }}$ the given analysis we studied various types of systems and problems concerning carbon nanoclusters. On the whole, the obtained results support using the fluctuation index $\sigma_{\text {IPR }}$ for interpreting localization phenomena in huge molecules and clusters.

As a conclusion, we can say that, in dealing with large orbital arrays of nanoclusters, the exhaustive description of their localization properties is a very difficult, if possible at all. Besides, we do not usually know beforehand possible regions of localization. In this situation, the proposed $\sigma_{\text {IPR }}$ index seems a useful interpretive tool. The index is quite sensitive to any spatial irregularity. Thus, it is enough to look at plot $\sigma_{\text {IPR }}$ vs eigenstate number, in order to trace orbitals with unusual localization properties. A successive study of few MOs thus detected is much more easy to perform. This procedure becomes practically needed in nanostructures, specially. In them 
one cannot restrict to studying, as usual, only few frontier MOs, because many MOs (scores and hundreds) can be active, having energies close to HOMO and LUMO energies.

Stress once more that the fluctuation index $\sigma_{\text {IPR }}$ serves as a direct indicator of the irregularity. Therefore, the orbitals with large values of this index may not be corresponding to a large localization on arbitrarily selected atom group or molecular fragment. The example of this kind was considered in Section 5. These situations are in fact inevitable since one can select many different fragments of molecule, while the MO with the extremal $\sigma_{\text {IPR }}=\sigma_{\text {IPR }}^{\max }$ is unique in fact. It should be recognized that in the complicated cases, orbital localizations are too involved, and additional global indicators are desirable for more exhaustible understanding.

A few words would be in order about how the proposed modification of orbital analysis can be extended to problems other than purely orbital ones. We have already discussed the EUE distribution $\left\{L_{A}^{\mathrm{EUE}}\right\}$ in terms localization indices (see Appendix). Using the same PR analysis for many-electron excited state seems even more attractive. However, only future concrete calculations will be able to tell us about a real use of such extensions of the presented interpretive tool.

Acknowledgments. The author is grateful to P.I.Belobrov and I.A.Denisov for the interesting discussion concerning the nanodiamond physics. This work was supported in part by the joint project of the National Academy of Sciences of Ukraine and the National Academy of Sciences of Belarus (Grant No. 09-06-16).

\section{Appendix. Localized states and effectively unpaired electrons}

The motivation for this appendix comes from the fact that there exist many physical manifestations of local frontier MOs in graphene networks. In particular, the intrinsic magnetism in them is closely related to the localized states [49-52]. In this context, the effectively unpaired electrons (EUE) are an important face of such magnetism. Here, following [21,34] we consider a simplistic interpretation of EUE in terms of Huckel orbitals and their localization.

Strictly speaking, in diamagnetic closedshell systems, EUE results from the electron correlation properly. The latter causes decoupling electrons with opposite spins, so in singlet states the electron pairing is partially lost. With this, virtual hole-particle pairs arise, and the fact can be expressed by an appropriate quantitative EUE measure which will be denoted by $N^{\mathrm{EUE}}$. As a possible definition for $N^{\mathrm{EUE}}$ the average population of the virtual hole-particle pairs was proposed [5,53]. This quite reasonable physical and simple definition is in essence equivalent to the well known Head-Gordon index [54], previously designed from formal analysis (for detail see [21,34] ). Our working expression is simply this:

$$
N^{\mathrm{EUE}}=2 \sum_{a} \lambda_{a}
$$

In Eq. (19), $\left\{\lambda_{a}\right\}$ are natural orbital occupation numbers for "virtual" part of 1-electron density matrix spectrum $(a \geq n+1$, and $n=N / 2$ where $N$ is an even number of electrons).

In the case of bipartite graphs (e.g., alternant hydrocarbons, graphenes, etc.) it is possible to construct the one-electron density matrix, which allows for a crude account of electron-correlation effects in terms of Huckel orbitals only ("quasi-correlation" model) [21]. It is based on a suitable approximation along the line of the different orbital for different spins theory. In the final formulation, an appropriate spin splitting parameter $\delta$ is introduced, and the virtual-orbital occupation numbers are directly estimated from the Huckel eigenspectrum $\left\{\varepsilon_{a}\right\}$, as follows:

$$
\lambda=1-\varepsilon_{a} / \sqrt{\delta^{2}+\varepsilon_{a}^{2}} .
$$

Besides, the EUE density matrix can be written down in terms of these $\lambda_{a}$ and Huckel MOs. As a result, the atomic localization indices $L_{A}^{\mathrm{EUE}}$ can be rather simply expressed, as follows:

$$
L_{A}^{\mathrm{EUE}}=2 \sum_{1 \leq i \leq n}\left(1-\left|\varepsilon_{i}\right| / \sqrt{\delta^{2}+\varepsilon_{i}^{2}}\right) L_{A}\left[\varphi_{i}\right],
$$

with orbital localizations $L_{A}\left(\varphi_{j}\right)$ computed by Eq. (4) from the occupied Huckel orbitals $\varphi_{i}$. With this, $\sum_{A} L_{A}^{\mathrm{EUE}}=N^{\mathrm{EUE}}$. The corresponding number of active atomic EUE centers is estimated as in Eq. (8):

$$
P R 2^{\mathrm{EUE}}=\left[\sum_{A}\left(L_{A}^{\mathrm{EUE}}\right)^{2}\right]^{2} / \sum_{A}\left(L_{A}^{\mathrm{EUE}}\right)^{4} .
$$


In agreement with [21, 34], Eqs. (A2)(A4) determine the basic results of the quasi-correlated tight-binding (QCTB) model for EUE.

After this preliminary, we can pass to a simplified study of EUE in the large graphene networks. We show in Table 5 the representative results for two structures from Table 2 (nanoribbon $\mathrm{C}_{228}$ and hexagonal $\mathrm{C}_{216}$ nanographene), and for two nanoparticles $\mathrm{C}_{1038}$ and $\mathrm{C}_{1200}$ which include pores - the so-called antidot structures. The latter became a rather frequent object of research scrutiny [55-57]. In all the systems studied, $N^{\mathrm{EUE}}$ reaches large values of (near 10). The PR2 $2^{\mathrm{EUE}}$ index reveals a rather delocalized EUE character, while having a considerable concentration of EUE at the edge (see $\left\{L_{A}^{\mathrm{EUE}}\right\}$ distributions in the Table). This fact can be treated qualitatively in terms of the frontier orbitals which give the essential contribution to $N^{\text {EUE }}$. Really, by restricting summation in Eq. (A3) to the first 10 virtual MOs, we obtain, say for $\mathrm{C}_{228}$ and $\mathrm{C}_{216}$, more than $60 \%$ of the whole $N^{\mathrm{EUE}}$ magnitude. Plots of the edge localization index $\left\{L_{\text {edge }}\left[\varphi_{j}\right]\right\}$ for occupied MOs (the last column of Table 5) tell us that only few frontier MOs are markedly localized on the egde, and just these frontier MOs provide a significant but not overwhelming contribution to EUE properties.

\section{References}

1. J.T.Edwards, D.J.Thouless, J.Phys.C, 5, 807 (1972); D.J.Thouless, Phys. Rep., 13, 93 (1974).

2. F.Evers, A.D.Mirlin, Rev.Mod.Phys., 80, 1355 (2008).

3. R.J.Bell, P.Dean, D.C.Hibbins-Butler, J.Phys. $C, 3,63$ (1970).

4. A.V.Luzanov, V.E.Umanski, Teor.Eksperim. Chem., 13, 162 (1977).

5. A.V.Luzanov, O.A.Zhikol, Int.J.Quantum Chem., 104, 167 (2005).

6. V.May, O.Kuhn, Charge and Energy Transfer Dynamics in Molecular Systems, Wiley-VCH, Weinheim (2011).

7. J.Pipek, G.Mezey, J.Chem.Phys., 90, 4916 (1989).

8. I.Tamm, Fiz.Zh. Soviet Union, 1, 733 (1932).

9. J.Koutecky, Adv. Chem. Phys., 9, 85 (1965).

10. S.G.Davison, J.D.Levine, Solid State Phys., 25, 32 (1970).

11. S.G.Davison, M.Steslicka, Basic Theory of Surface States, Clarendon Press, Oxford (1996).

12. K.Sattler, in: Handbook of Thin Film Materials, v.5, ed. by H.S.Nalwa, Academic, New York (2002), p.61.
13. Y.-W.Son, M.L.Cohen, S.G.Louie, Nature, 444, 347 (2006).

14. M.Nishida, J.Appl. Phys., 104, 086101 (2008).

15. G.W.Bryant, J.Comput.Theor. Nanosci., 6, 1262 (2009).

16. L.Jiang, Y.Zheng, C.Yi et al., Phys.Rev.B, 80, 155454 (2009)

17. H.M.Luhavaya, M.V.Pavlov, A.Yu.Ermilov, N.F.Stepanov, Zh. Fiz. Khim.A, 86, 1261 (2012).

18. I.A.Denisov, A.A.Zimin, L.A.Bursill, P.I.Belobrov, J.Sib.Fed.Univ. Math.Phys., 7, 3 (2014).

19. O.-A.Dobrescu, M.Apostol, Can.J.Phys., 93, 580 (2015).

20. A.V.Luzanov, J. Struct. Chem., 55, 799 (2014); Functional Materials, 22, 514 (2015).

21. A.V.Luzanov, Functional Materials, 21, 437 (2014).

22. N.C.Murphy, R.Wortis, W.A.Atkinson, Phys. Rev. B, 83, 184206 (2011).

23. J.E.Lennard-Jones, Proc.Roy.Soc.A, 158, 280 (1937).

24. I.S.Gradshteyn, I.M.Ryzhik, Table of Integrals, Series, and Products, Academic, San Diego, CA (2007).

25. G.F.Kventsel, Teor. Eksper. Khim., 5, 287 (1972).

26. S.E.Stein, R.L.Brown, Carbon, 23, 105 (1985); J.Am.Chem.Soc., 109, 3721 (1987).

27. K.Nakada, M.Fujita, G.Dresselhaus, M.S.Dresselhaus, Phys. Rev.B, 54, 17954 (1996).

28. M.Fujita, K.Wakabayashi, K.Nakada, K.Kushakabe, J.Phys.Soc.Jpn., 65, 1920 (1996).

29. T.Enoki, Y.Kobayashi, K.Fukui, Int.Rev. Phys.Chem., 26, 609 (2007).

30. M.Vanevic, V.M.Stojanovi, M.Kindermann, Phys.Rev. B, 80, 045410 (2009).

31. Y.Kobayashi, K.Fukui, T.Enoki et al., Phys. Rev. B, 71, 193406 (2005).

32. Y.Niimi, T.Matsui, H.Kambara et al., Phys. Rev. B, 73, 085421 (2006).

33. K.Sugawara, T.Sato, S.Souma et al., Phys. Rev. B, 73, 045124 (2006).

34. A.V.Luzanov, in: Practical Aspects of Computational Chemistry IV, ed. by J.Leszczynski, M.K.Shukla, Springer, New York (2016).

35. Physics and Applications of CVD Diamond, ed. by C.Nebel, M.Nesladek, Wiley, Weinheim, p. 151 (2008).

36. A.Kruger, Carbon Materials and Nanotechnology, Wiley-VCH, Weinheim (2010).

37. V.Georgakilas, J.A.Perman, J.Tucek, R.Zboril, Chem. Rev., 115, 4744 (2015).

38. A.V.Luzanov, O.A.Zhikol, I.V.Omelchenko et al., Functional Materials, in press.

39. R.Hoffmann, J.Chem.Phys., 39, 1397 (1963).

40. A.V.Luzanov, O.A.Zhikol, Functional Materials, 23, 63 (2016).

41. A.Konishi, T.Kubo, in: Chemical Science of p-Electron Systems, ed. by T.Akasaka, A.Osuka, S.Fukuzumi et al., Springer, Japan (2015), p.337.

42. O.Ivanciuc, D.J.Klein, L.Bytautas, Carbon, 40, 2063 (2002). 
43. W.Jaskolski, A.Ayuela, M.Pelc et al., Phys. Rev. B, 83, 235424 (2011).

44. E K.Fujisawa, R.Cruz-Silva, K.-S.Yang et al., J. Mater. Chem. A, 2, 9532 (2014).

45. F.London, J.Phys. Radium, 8, 397 (1937); T.E.Peacock, Electronic Properties of Aromatic and Heterocyclic Molecules, Academic, London (1965).

46. T.E.Stacey, D.C.Fredrickson, Dalton Trans., 41, 7801 (2012).

47. N.A.Popov, J.Struct. Chem., 11, 670 (1971).

48. P.W.Fowler, E.Stainer, Chem. Phys. Lett., 364, 259 (2002).

49. K.Wakabayashi, M.Fujita, H.Ajiki, M.Sigrist, Phys. Rev. B, 59, 8271 (1999).
50. K.Kusakabe, M.Maruyama, Phys.Rev.B, 67, 092406 (2003).

51. J.Fernandez-Rossier, J.J.Palacios, Phys.Rev. Lett., 99, 177204 (2007).

52. O.V.Yazyev, Rep.Prog.Phys., 73, 056501 (2010).

53. A.V.Luzanov, O.V.Prezhdo, J.Chem.Phys., 125, 154106 (2006).

54. M.Head-Gordon, Chem.Phys. Lett., 372, 508 (2003).

55. T.Pedersen, C.Flindt, J.Pedersen et al., Phys. Rev. Lett., 100, 136804 (2008).

56. LP.Biro, P.Nemes-Incze, P.Lambin, Nanoscale, 4, 1824 (2012).

57. M.Wierzbicki, R.Swirkowicz, J.Barnas, Phys. Rev., 88, 235434 (2013). 